\title{
The Effect of Lactate Level on Early Mortality and Late Wound Healing in Children with Thoracoabdominal Trauma with Fluid Treatment Before Hospital
}

\author{
Cengiz Güney ${ }^{1}$, Abuzer Coskun²,*
}

${ }^{1}$ Department of Pediatric Surgery, Cumhuriyet University Medical Faculty, Sivas, Turkey

${ }^{2}$ SBU Istanbul Bağcılar Training and Research Hospital, Emergency Medicine Clinic, Istanbul, Turkey

\section{*Correspondence}

dr.acoskun44@hotmail.com

(Abuzer Coskun)

\begin{abstract}
Background: In this study, we aimed to evaluate the effect of serum lactate levels on early mortality and late wound healing in patients with childhood thoracoabdominal trauma, pre-hospital fluid replacement in patients admitted to the emergency department. Methods: This study included 479 patients under the age of 18 who applied to the emergency room for thoracoabdominal trauma between January 2014 and December 2018. Of these, $278(58 \%)$ were male, with a mean age of 8.34 years (range, 1-16 years). Demographic characteristics, fluid resuscitation, and serum lactate levels, trauma pattern, and mortality results of these patients were evaluated retrospectively. Results: $474(98.9 \%)$ of the cases were blunt and $5(1.1 \%)$ were penetrating trauma. $225(47 \%)$ of the trauma cases were less than one meter falling, 162 (33.8\%) were over one meter falling from the high, and 87 (18.2\%) were car accidents inside and outside. Mortality was found most common in falling from height cases, which were related to prolonged hospitalization and high lactate level $(\mathrm{p}<0.05)$. Wound healing times of patients undergoing pre-hospital fluid resuscitation and wound healing times of patients without pre-hospital fluid resuscitation were compared. Multivariate regression analysis, lactate level, and wound healing time were found to be predictive of the values found to be significant in the univariate regression analysis with fluid resuscitation and mortality ( $p$ $<0.05$ ). Conclusion: Most thoracoabdominal traumas can be treated with conservative methods. Pre-hospital fluid treatment in severe trauma cases may lead to decreased lactate levels, reduced mortality, and shortened wound healing time.
\end{abstract}

\section{Keywords}

Emergency, Child thoracoabdominal trauma, Fluid resuscitation, Lactate, Wound healing, Mortality

\section{Introduction}

One of the preventable causes of death within the first 24 hours after trauma is responsible for $30-40 \%$ of trauma mortality [1]. The general approach is to perform intravenous fluid replacement at the trauma site and during the patient's transfer. However, there are reports that this practice may not be the right approach in all trauma patients [2,3]. In some studies, it is believed that patients' time to reach the hospital is increased and the risk of death increases due to intravenous access and liquid replacement during the on-site transfer and transfer [46]. It has been reported that pre-hospital fluid administration in penetrating trauma may increase bleeding and mortality, thus delaying fluid resuscitation may be appropriate in these patients [7]. The number of patients with blunt trauma remains limited in this regard [8].

Lactate, a by-product of glycolysis, is produced in many organs. It is eliminated by the liver, kidneys, and muscles. It is the first time, Broder et al. reported that lactate level was associated with poor prognosis in patients with shock [9]. During trauma ischemia due to hemorrhagic shock causes insufficient oxygen supply, metabolic acidosis, and deficiency of oxygen. The association between increased lactate levels due to tissue hypoxia and wound healing has been reported previously $[10,11]$. Lactate measurements in arterial blood gas can be used as a determinant of tissue hypoxia and metabolic acidosis after trauma. Serial lactate measurements may also be useful in predicting mortality in trauma patients [12].

In this study, we aimed to investigate the effect of prehospital fluid resuscitation on serum lactate levels, mortality in the early period and wound healing in the late period among pediatric thoracoabdominal trauma patients. 
TA B L E 1. Baseline characteristics of study patients.

Fluid Resuscitation

All patients Patients with

\begin{tabular}{|c|c|c|c|c|c|}
\hline & All patients & Patients with & & & \\
\hline & & No & Yes & $\mathbf{z}$ & p-value \\
\hline \multicolumn{6}{|c|}{ Basaline Characteristcis } \\
\hline Age, mean \pm SD, yr & $8.34 \pm 3.28$ & $8.23 \pm 3.31$ & $8.44 \pm 3.25$ & -0.494 & 0.621 \\
\hline Sex, Female/Male & $201 / 278$ & $111 / 131$ & $90 / 147$ & $X^{2}=3.069$ & 0.080 \\
\hline PRT, day & $16.47 \pm 4.11$ & $19.48 \pm 2.91$ & $13.40 \pm 2.62$ & -17.066 & 0.001 \\
\hline \multicolumn{6}{|l|}{ Labaratory Finding } \\
\hline \multicolumn{6}{|l|}{ Biochemistry } \\
\hline BUN, mg/dL & $20.93 \pm 9.51$ & $25.98 \pm 9.74$ & $15.76 \pm 5.78$ & -11.972 & 0.001 \\
\hline Krea, mg/dL & $0.80 \pm 0.27$ & $0.92 \pm 0.29$ & $0.68 \pm 0.18$ & -9.401 & 0.001 \\
\hline ALT, mg/dL & $28.21 \pm 17.36$ & $38.00 \pm 19.10$ & $18.20 \pm 6.26$ & -13.832 & 0.001 \\
\hline AST, mg/dL & $32.72 \pm 20.90$ & $45.78 \pm 21.30$ & $19.40 \pm 8.28$ & -14.633 & 0.001 \\
\hline ALP, mg/dL & $97.76 \pm 49.75$ & $98.30 \pm 50.44$ & $97.22 \pm 49.13$ & -0.300 & 0.976 \\
\hline BS, mg/dL & $122.57 \pm 29.58$ & $133.57 \pm 33.17$ & $111.33 \pm 19.93$ & -8.127 & 0.001 \\
\hline \multicolumn{6}{|l|}{ Hemogram } \\
\hline WBC, $\mathrm{mg} / \mathrm{dL}$ & $10.04 \pm 3.86$ & $9.68 \pm 3.74$ & $10.41 \pm 3.95$ & -2.010 & 0.044 \\
\hline $\mathrm{Hb}, \mathrm{g} / \mathrm{dL}$ & $13.86 \pm 1.89$ & $13.74 \pm 1.80$ & $13.97 \pm 1.97$ & -1.629 & 0.103 \\
\hline Hct, \% & $41.42 \pm 6.21$ & $40.95 \pm 6.06$ & $41.90 \pm 6.34$ & -2.054 & 0.040 \\
\hline \multicolumn{6}{|l|}{ ABG } \\
\hline $\mathbf{N a}^{+}, \mathrm{mmol} / \mathrm{L}$ & $138.18 \pm 4.91$ & $138.40 \pm 5.28$ & $137.82 \pm 4.49$ & -0.703 & 0.482 \\
\hline $\mathbf{K}^{+}, \mathrm{mmol} / \mathrm{L}$ & $3.88 \pm 0.70$ & $4.00 \pm 0.76$ & $3.74 \pm 0.61$ & -4.110 & 0.001 \\
\hline $\mathbf{C a}^{++}, \mathrm{mmol} / \mathrm{L}$ & $1.04 \pm 0.24$ & $1.05 \pm 0.25$ & $1.02 \pm 0.23$ & -1.785 & 0.074 \\
\hline Lac, mmol/L & $2.12 \pm 1.51$ & $2.86 \pm 1.71$ & $1.38 \pm 0.74$ & -17.066 & 0.001 \\
\hline Mortality & & No & Yes & & \\
\hline \multirow[t]{2}{*}{ HT, day } & & $16.26 \pm 3.94$ & $21.91 \pm 4.69$ & -4.599 & 0.001 \\
\hline & & $1.95 \pm 1.20$ & $6.64 \pm 1.89$ & -6.930 & 0.001 \\
\hline
\end{tabular}

Yr: year, PRT: Posttraumatic Recovery Time, BUN: Blood Urea Nitrogen, Krea: Kreatinin, ALT: Alanin Aminotransferaz, AST: Aspartat Aminotransferaz, ALP: Alkalen Fosfataz, BS: Blood Sugar, WBC: White Blood Cell, $\mathrm{Hb}$ : Hemoglobin, Hct: Hematocrit, $\mathrm{ABG}$ : Arterial blood gas $\mathrm{Na}^{+}$: Sodium, $\mathrm{K}^{+}$: Potassium, $\mathrm{Ca}^{++}$: Calcium,

Lac: Lactate, HT: Hospitalization time.

\section{Materials and methods}

\subsection{Study design and population}

This study was performed retrospectively. 479 patients under 18 years of age who applied to Sivas Cumhuriyet University and Sivas Numune Hospital Emergency Services between January 2014 and December 2018 and who had thoracoabdominal trauma were included. After the etiologic trauma factors, organ involvement, and classification were determined, the patients were hospitalized in the pediatric surgery clinic.

Exclusion cases from the study were determined as in the following. 1- the causes of trauma other than the first 24 hours, 2 - the plain radiographs required for the diagnosis, thoracic and abdominal computed tomographies and/or ultrasonography of patients, 3- gunshot wounds, 4- small house accidents, 5incisions.

Demographic features, trauma types, examination results, thoracoabdominal traumas, other organ injuries, fluid resus- citation, and serum lactate level results were recorded.

Patients were divided into two groups according to fluid resuscitation. Fluid resuscitation of these patients were obtained from the Emergency Medical System Ambulance Services records. After the patients were discharged after the trauma, they were followed-up for six months with the automation system. During this period, patients without access were called by phone and the recovery period was questioned. Diagnoses, admission dates, contact information, and demographic, clini$\mathrm{cal}$, and laboratory data are included in the registration system of our hospital. As a result, all of the patients were reached via phone calls and/or hospital records.

Trauma cases divided into five groups as below. 1-falls down to one meter "generally fall from places such as swing, bicycle, stairs, and walls not higher than one meter, bunk beds," 2-drops from one meter and above, 3-in-car traffic accidents, 4-outside vehicle traffic accidents, 5-piercing-cutting tool injuries. 
TA B L E 2. Chi-square test of trauma types with variables.

\begin{tabular}{|c|c|c|c|c|c|c|c|c|}
\hline \multicolumn{9}{|c|}{ Types of Trauma } \\
\hline & & $-1 m F n(\%)$ & $+1 \mathrm{mD} n(\%)$ & ICTA n(\%) & NCTA n(\%) & PCTI n(\%) & $\chi 2$ & p-value \\
\hline \multirow[t]{2}{*}{ Gender } & Female & 89(18.6) & $72(13.5)$ & $21(4.4)$ & $16(3.3)$ & $3(0.6)$ & 1.629 & 0.804 \\
\hline & Male & $136(28.4)$ & $90(18.8)$ & $28(5.8)$ & $22(4.6)$ & $2(0.4)$ & & \\
\hline \multirow[t]{2}{*}{ Thoracic V } & No & $224(46.8)$ & $159(33.2)$ & $47(9.8)$ & $35(7.3)$ & $5(1)$ & 11.357 & 0.001 \\
\hline & Yes & $1(0.2)$ & $3(0.6)$ & $2(0.4)$ & $3(0.6)$ & $0(0)$ & & \\
\hline \multirow[t]{2}{*}{ Lumbal V } & No & $223(46.6)$ & $156(32.6)$ & $45(9.4)$ & $34(7.1)$ & $5(1)$ & 14.035 & 0.007 \\
\hline & Yes & $2(0.4)$ & $6(1.3)$ & $4(0.8)$ & $4(0.8)$ & $0(0)$ & & \\
\hline \multirow[t]{2}{*}{ Lungs } & No & $215(44.9)$ & $136(28.4)$ & $37(7.7)$ & $24(5)$ & $3(0.6)$ & 42.872 & 0.001 \\
\hline & Yes & $10(2.1)$ & $26(5.4)$ & $12(2.5)$ & $14(2.9)$ & $2(0.4)$ & & \\
\hline \multirow[t]{2}{*}{ Rib fracture } & No & $220(45.9)$ & $142(29.6)$ & $41(8.6)$ & $33(6.9)$ & $5(1)$ & 20.943 & 0.001 \\
\hline & Yes & $5(1)$ & $20(4.2)$ & $8(1.7)$ & $5(1)$ & $0(0)$ & & \\
\hline \multirow[t]{2}{*}{ Px } & No & $223(46.6)$ & $156(32.6)$ & $45(9.4)$ & $34(7.1)$ & $5(1)$ & 14.035 & 0.007 \\
\hline & Yes & $2(0.4)$ & $6(1.3)$ & $4(0.8)$ & $4(0.8)$ & $0(0)$ & & \\
\hline \multirow[t]{2}{*}{$\mathbf{H x}$} & No & $223(46.6)$ & 161(33.6) & $44(9.2)$ & $36(7.5)$ & $5(1)$ & 21.068 & 0.001 \\
\hline & Yes & $2(0.4)$ & $1(0.2)$ & $5(1)$ & $2(0.4)$ & $0(0)$ & & \\
\hline \multirow[t]{2}{*}{ IAB } & No & $223(46.6)$ & $155(32.4)$ & $41(8.6)$ & $31(6.5)$ & $4(0.8)$ & 36.610 & 0.001 \\
\hline & Yes & $2(0.4)$ & $7(1.5)$ & $8(1.7)$ & $7(1.5)$ & $1(0.2)$ & & \\
\hline \multirow[t]{2}{*}{ IAOI } & No & $222(46.3)$ & $151(31.5)$ & $37(7.7)$ & $32(6.7)$ & $3(0.6)$ & 46.399 & 0.001 \\
\hline & Yes & $3(0.6)$ & 11(2.3) & $12(0.6)$ & $6(1.3)$ & $2(0.4)$ & & \\
\hline \multirow[t]{2}{*}{ FR } & No & $106(22.1)$ & $84(17.5)$ & $27(5.6)$ & $22(4.6)$ & $3(0.6)$ & 2.579 & 0.631 \\
\hline & Yes & $119(24.8)$ & $78(16.3)$ & $22(4.6)$ & $16(3.3)$ & $2(0.4)$ & & \\
\hline \multirow[t]{2}{*}{ Mortality } & No & $222(46.3)$ & $153(31.9)$ & $48(10)$ & $34(7.1)$ & $4(0.8)$ & 13.965 & 0.007 \\
\hline & Yes & $3(0.6)$ & $9(1.9)$ & $1(0.2)$ & $4(0.8)$ & $1(0.2)$ & & \\
\hline
\end{tabular}

-1mF: Falls up to one meter, $+1 \mathrm{mD}$ : Drops from a meter and more, ICTA: In-car traffic accident, NCTA: Nonvehicle traffic accident, PCTI: Penetrating-cutting tool injury, V: Vertebrae, Px: Pneumothorax, Hx: Hemothorax, IAB: Intraabdominal bleeding, IAOI; Intra-abdominal organ injury; FR: Fluid Resuscitation.

Hemogram was measured using a Beckman Coulter Automated CBC Analyzer (Beckman Coulter, Inc., Fullerton, CA, USA).

Biochemistry blood was analyzed with the Cobas 6000 (C6000-Core, Cobas c-501 series, Hitachi, Roche, USA).

The patients' lactate levels were obtained from arterial blood gas analyses using the Acobas $₫$ b221 Blood Gas system (Roche, Basel, Switzerland).

Organ injuries were determined by imaging methods (ultrasonography, computed tomography, direct radiography).

\subsection{Statistical Analyses}

The obtained data from this study were analyzed with SPSS 20 software (IBM SPSS Statistics Software). While investigating variables from a normal distribution, Shapiro Wilk's was used due to the number of units. Descriptive statistics were shown as mean \pm standard deviation or median (minimum-maximum) for continuous variables, and nominal variables were shown in several cases and percentages (\%). A Chi-square test was applied to examine the relationship between nominal variables among the groups. If the predicted values in the cells of $2 \times 2$ tables did not have enough volume, Fisher's exact test was used, and the Pearson Chi-square test was used with the help of Monte Carlo Simulation to analyze $\mathrm{R} \times \mathrm{C}$ tables. Also, linear regression was used for the univariate and multivariate analyses of the variables. Univariate analysis was used to measure the relationship of variables, by the development of trauma types. The variables that were found to be statistically significant in the univariate analysis were used in the multivariate linear regression risk model with the forward stepwise method to determine the independent prognostic factor for the development of trauma varieties. When interpreting the results, values below $\mathrm{p}<0.05$ were considered statistically significant.

\section{Results}

In this study, 479 patients were evaluated who are admitted to the emergency room due to thoracoabdominal trauma between January 2014 and December 2018. The average age of the patients was $8.34 \pm 3.28$ /year the male-female ratio was 1.38 . $59.1 \%$ of the patients were male and $41.9 \%$ were female (Table 1). The number of patients who underwent fluid replacement before being brought to the emergency room was 237 (49.4\%) and the male/female ratio was 1.63 . The demographic charac- 
TA B L E 3. Chi-square test analysis according to the variables of mortality.

\begin{tabular}{|c|c|c|c|c|c|}
\hline & & \multicolumn{4}{|l|}{ Mortality } \\
\hline & & No n(\%) & Yes n(\%) & $\chi^{2}$ & p-value \\
\hline \multirow[t]{2}{*}{ Gender } & Female & $195(40.7)$ & $6(1.3)$ & 0.572 & 0.45 \\
\hline & Male & $266(55.5)$ & $12(2.5)$ & & \\
\hline \multirow[t]{2}{*}{ Thoracic V } & No & $453(94.6)$ & $17(3.5)$ & 1.371 & 0.242 \\
\hline & Yes & $8(1.7)$ & $1(0.2)$ & & \\
\hline \multirow{2}{*}{ Lumbal V } & No & $447(93.3)$ & $16(3.3)$ & 3.498 & 0.061 \\
\hline & Yes & $14(2.9)$ & $2(0.4)$ & & \\
\hline \multirow[t]{2}{*}{ Lungs } & No & $399(83.3)$ & $16(3.3)$ & 0.082 & 0.775 \\
\hline & Yes & $62(12.9)$ & $2(0.4)$ & & \\
\hline \multirow[t]{2}{*}{ Rib fracture } & No & $425(88.7)$ & $16(3.3)$ & 0.259 & 0.611 \\
\hline & Yes & $36(7.5)$ & $2(0.6)$ & & \\
\hline \multirow[t]{2}{*}{ Px } & No & $447(93.3)$ & $16(3.3)$ & 3.498 & 0.061 \\
\hline & Yes & $14(2.9)$ & $2(0.4)$ & & \\
\hline \multirow[t]{2}{*}{$\mathbf{H x}$} & No & $452(94.4)$ & $17(3.5)$ & 1.100 & 0.294 \\
\hline & Yes & $9(1.9)$ & $1(0.2)$ & & \\
\hline \multirow[t]{2}{*}{ IAB } & No & $439(91.6)$ & $15(3.1)$ & 4.955 & 0.026 \\
\hline & Yes & $22(4.6)$ & $3(0.6)$ & & \\
\hline \multirow[t]{2}{*}{ IAOI } & No & $431(90)$ & $14(2.9)$ & 6.488 & 0.011 \\
\hline & Yes & $30(6.3)$ & $4(0.8)$ & & \\
\hline \multirow[t]{2}{*}{ FR } & No & $227(47.4)$ & $15(3.1)$ & 8.055 & 0.005 \\
\hline & Yes & $234(48.9)$ & $3(0.6)$ & & \\
\hline \multirow[t]{5}{*}{ Trauma } & $-1 \mathrm{mF}$ & $222(46.3)$ & $3(0.6)$ & 13.965 & 0.007 \\
\hline & $+1 \mathrm{mD}$ & 153(31.9) & $9(1.9)$ & & \\
\hline & ICTA & $48(10)$ & $1(0.2)$ & & \\
\hline & NCTA & $34(7.1)$ & $4(0.6)$ & & \\
\hline & PCTI & $4(0.8)$ & $1(0.2)$ & & \\
\hline
\end{tabular}

teristics of the patients, the averages of arterial blood gas, and biochemical values were shown in Table 1.

Wound healing times of patients who had pre-hospital fluid resuscitation were shorter compared to without pre-hospital fluid resuscitation and were found statistically significant $(\mathrm{p}$ $<0.05)$.

The patients were evaluated in terms of biochemical, hematological, and arterial blood gas parameters. Blood urea nitrogen (BUN), creatinine (Cre), alanine aminotransferase (ALT), aspartate aminotransferase (AST), blood sugar (BS), white blood cell (WBC), hematocrit (Hct), blood gas values were found statistically significant differences between fluid replacement group $(\mathrm{p}<0.05$, Table 1$)$.

The relationship between arterial blood gas lactate levels and mortality was evaluated, the average of lactate level in the group with mortality was high and low in the group without mortality $(p<0.05)$. The relationship between blood gas lactate levels and hospitalization period of patients with mortality was examined, it was seen that there was a statistically significant difference $(\mathrm{p}<0.05)$. In the group with mortality, the mean life expectancy of patients who underwent Prehospital fluid resuscitation (PHFR) was calculated longer (NonPHFR:
$16.26 \pm 3.94$ days, PHFR: $21.91 \pm 4.69$ days $)(\mathrm{p}<0.05$, Table 1).

There was no statistically significant difference in the distribution of trauma types by gender $(p>0.05)$. Similarly, the distribution of patients with PHFR and NonPHFR according to trauma types did not differ statistically $(p>0.05)$. This situation is valuable for showing that the distribution of our sample according to trauma types is homogeneous (Table 2).

There was no significant difference in the relationship between mortality and gender $(p=0.450)$. There was no statistically significant relationship between pulmonary contusion with thoracic pathologies $(p>0.05)$, rib fracture, hemothorax and pneumothorax, and the relationship between intraabdominal pathologies and mortality was statistically significant $(\mathrm{p}$ $<0.05$ ). There was a statistically significant relationship between pre-hospital fluid resuscitation and mortality. The mortality rates were significantly high in patients of The NonPHRF group ( $p<0.05$, Table 3$)$.

In terms of fluid resuscitation and mortality, data that were statistically significant compared to univariate cox regression were determined as a predictive value for lactate and posttraumatic recovery time (PRT) in multivariate cox regression 
TA B L E 4. Univariate and multivariate Cox regression analyses for predicting the development of Fluid Resuscitation and Mortality.

\begin{tabular}{|c|c|c|c|c|c|c|c|c|}
\hline \multicolumn{9}{|c|}{ Fluid Resuscitation } \\
\hline \multicolumn{3}{|c|}{ Univariate } & \multicolumn{3}{|c|}{ Multivariate } & \multicolumn{3}{|c|}{ Correlation } \\
\hline & HR & $95 \% \mathrm{Cl}$ & p-value & HR & $95 \% \mathrm{Cl}$ & p-value & $\mathbf{r}$ & p-value \\
\hline BUN & 0.845 & $0.818-0.874$ & 0.001 & 0.895 & $0.831-0.963$ & 0.003 & 0.548 & 0.001 \\
\hline Kreatinin & 0.013 & $0.005-1.035$ & 0.035 & 0,004 & $0.000-0.078$ & 0.001 & -0.43 & 0.001 \\
\hline BS & 0.966 & $0.957-0.975$ & 0.001 & 0.946 & $0.923-0.969$ & 0.001 & -0.372 & 0.001 \\
\hline Lac & 0.043 & $0.022-0.083$ & 0.001 & 0.173 & $0.062-0.461$ & 0.001 & -0.781 & 0.001 \\
\hline PRT & 0.476 & $0.419-0.540$ & 0.001 & 0.612 & $0.490-0.763$ & 0.001 & -0.759 & 0.001 \\
\hline Mortality & 0.194 & $0.055-0.679$ & 0.010 & 3.551 & $2.303-6.085$ & 0.023 & -0.13 & 0.004 \\
\hline ALT & 0.854 & $0.830-0.878$ & 0.001 & & & & -0.633 & 0.001 \\
\hline AST & 0.877 & $0.857-0.897$ & 0.001 & & & & -0.669 & 0.001 \\
\hline WBC & 1.051 & $1.002-1.102$ & 0.040 & & & & 0.092 & 0.044 \\
\hline $\mathbf{K}^{+}$ & 0.578 & $0.440-0.760$ & 0,001 & & & & -0.188 & 0.001 \\
\hline $\mathbf{N a}^{+}$ & 0.976 & $0.940-1.012$ & 0.191 & & & & -0.032 & 0.483 \\
\hline ALP & 1 & $0.996-1.003$ & 0.811 & & & & -0.001 & 0.976 \\
\hline $\mathbf{C a}^{++}$ & 0.559 & $0.265-1.183$ & 0.128 & & & & -0.082 & 0.074 \\
\hline Age & 1.02 & $0.966-1.077$ & 0.48 & & & & 0.023 & 0.622 \\
\hline \multirow[t]{2}{*}{ Gender } & 1.384 & 0.961-1.992 & 0.08 & & & & -0.023 & 0.411 \\
\hline & Mortalit & & & & & & & \\
\hline Lac & 2.499 & $1.909-3.270$ & 0.001 & 3.762 & $1.811-7.813$ & 0.001 & 0.317 & 0.001 \\
\hline PRT & 1.446 & $1.248-1.675$ & 0.001 & 1.415 & $1.063-1.884$ & 0.017 & 0.21 & 0.001 \\
\hline
\end{tabular}

Multiple Cox proportional hazards model includes all the variables in univariate analysis with forward stepwise method. Abbreviations: CI, confidence interval; HR, hazard ratio.

analysis. Also, these variables were found to be a significant relationship with strong negative correlations (Table 4).

\section{Discussion}

In our study, the relationship between mortality and late wound healing of traumatic patients with or without pre-hospital fluid resuscitation (PHRF) and arterial blood gas lactate levels were investigated.

There are many contradictory studies in the literature on the effect of pre-hospital fluid resuscitation. The number of studies with evidence of increased mortality in patients undergoing PHFR with penetrating trauma is increasing in recent years $[7,13]$. However, since the mechanism of penetrating trauma and the mechanism of blunt trauma are different from each other, it may be expected that PHFR may be beneficial in patients with blunt trauma [14]. Mizushima et al [8]. suggested that a restricted PHFR may be useful. On the other hand, it has been predicted that the aggressive application of pre-hospital intravenous fluid therapy may cause the opening of clots resulting from elevated blood pressure and cause recurrent bleeding $[13,14]$. Therefore, limited fluid treatment is recommended only in hypotensive patients.

Sammour et al. has been reported that a significant relationship between serum lactate level and trauma scores (ISS, injury severity score; TRISS, trauma, and injury severity score) in adult trauma patients [15]. In our study, the relationship between pre-hospital fluid therapy and serum lactate level with wound healing and mortality was investigated. However, in this study, the effectiveness of prehospital fluid therapy was not examined.

In our study, $242(50.5 \%)$ of 479 trauma patients were not treated with PHRF. The mean PRT of these patients was 19.48 \pm 2.91 , whereas the PRT of the patients who underwent PHRF was $13.4 \pm 2.62$. The PRT of patients undergoing pre-hospital fluid resuscitation was found to be shorter and more significant compared to patients who did not undergo fluid resuscitation. Our study is important in terms of demonstrating that PHRF administration may be beneficial for the PRT abbreviation in trauma patients. In patients receiving PHRF, shortening the wound healing time compared to NonPHRF patients was found to be predictive. Mortality was observed in $1.26 \%$ of patients undergoing pre-hospital fluid resuscitation and $6.19 \%$ of NonPHRF patients. A significant difference in mortality between two different applications could be an important parameter in the studies of fluid resuscitation and serum lactate level.

Serum lactate is produced as a result of anaerobic metabolism and is a marker of inadequate tissue perfusion. Increased complication and mortality are reported in adult patients with clinically no symptoms of shock but with high serum lactate levels $[16,17]$. At the same time, the symptoms of shock and organ dysfunction of the high lactate level in 
sepsis patients are associated with high mortality even without clinical presentation [12]. Uzoigwe et al. has been reported that the lactate level may be a prognostic criterion in predicting latent hypoperfusion and the outcome of trauma patients [18].

In our study, the level of lactate in patients undergoing PHRF was significantly lower than n-PHRF. The lactate level of 18 patients with mortality was $6.64 \pm 1.89 \mathrm{mmol} / \mathrm{L}$ and this level was $1.95 \pm 1.20 \mathrm{mmol} / \mathrm{L}$ in patients without mortality. The application of PHRF, which limits the elevation of the Lactate level, is an important parameter for demonstrating the improvement of tissue perfusion. In this study, the significant relationship was determined between the lactate level and mortality rate in the univariate and multivariate Cox regression method in terms of the effect of PHRF. A similar study was conducted by Lewis et al. pre-hospital the lactate measurements can be used as a marker for predicting mortality and therefore portable lactate measuring devices can be carried by first aid teams [19]. We believe that the use of mobile devices that can measure the lactate level will be important in determining which patients should undergo PHRF. Balanced fluid therapy in patients with hypotensive and high lactate levels may improve survival in trauma patients. At the same time, the measurement of lactate levels is important in that it may be beneficial to administer fluid therapy in patients.

\section{Conclusion}

It is important to increase survival in trauma patients which are the most major cause of mortality in the young age group. In recent years, when pre-hospital fluid resuscitation is controversial, it will improve the survival rates by preventing erroneous decisions by looking at the lactate level values. PHRF administration significantly decreases mortality in patients with increased prehospital lactate levels. It was observed that prehospital fluid resuscitation decreased serum lactate level and mortality and decreased serum lactate level also shortened wound healing time. Fluid treatment for patients with high lactate levels with a pre-hospital portable blood gas device may contribute to reducing the mortality rate by shortening wound healing and hospital stay.

\section{STUDY LIMITATION}

The most important limitation of the study is that it is retrospective and single centered. Also, it was important to determine the recovery time by calling the patients after the hospital, difficulty in accessing the records, and giving and receiving pre-hospital fluids in the emergency medical system.

\section{ACKNOWLEDGEMENTS}

Thanks to all the peer reviewers and editors for their opinions and suggestions.

\section{CONFLICT OF INTEREST}

The authors have no conflict of interest to declare.

\section{INFORMED CONSENT}

Written informed consent was not necessary because the study was performed retrospectively by screening patient files.

\section{ETHICAL APPROVAL}

The study was approved by the Local Ethics Committee of Cumhuriyet University Faculty of Medicine (date: 17.04.2019, no: 04/07).

\section{AUTHORSHIP CONTRIBUTIONS}

Concept: A.C., C.G.; Design: A.C.; Supervision: C.G.; Fundings: A.C; Materials: C.G; Data: A.C; Analysis: A.C., C.G.; Literature search: A.C.; Writing: A.C., C.G; Critical revision: A.C.

\section{AVAILABILITY OF DATA AND MATERIALS}

All data is available on request without restriction.

\section{HUMAN RIGHTS}

The study was made in following the Declaration of Helsinki for Human Research.

\section{FINANCIAL DISCLOSURE}

The authors declared that this study has received no financial support.

\section{REFERENCES}

[1] Holcomb JB, Wade CE, Trauma Outcomes Group, et al. Trauma Outcomes Group. Defining present blood component transfusion practices in trauma patients: papers from the Trauma Outcomes Group. J Trauma. 2011;71:315-317.

[2] Seamon MJ, Fisher CA, Gaughan J, et al. Prehospital procedures before emergency department thoracotomy: "Scoop and Run" saves lives. J Trauma Acute Care Surg. 2007;63:113-120.

[3] Krausz MM, Bar-Ziv M, Rabinovici R, et al. "Scoop and Run" or stabilize hemorrhagic shock with normal saline or small-volume hypertonic saline? J Trauma Acute Care Surg. 1992;33:6-10.

[4] Kauvar DS, Lefering R, Wade CE. Impact of hemorrhage on trauma outcome: an overview of epidemiology, clinical presentations, and therapeutic considerations. J Trauma. 2006;60:3-11.

[5] Salomone JP, Ustin JS, McSwain NE Jr, et al. Opinions of trauma practitioners regarding prehospital interventions for critically injured patients. J Trauma. 2005;58:509-515.

[6] Trunkey DD. Is ALS necessary for pre-hospital trauma care? J Trauma. 1984;24:86-87.

[7] Bickell WH, Wall MJ, Pepe PE, et al. Immediate versus delayed fluid resuscitation for hypotensive patients with penetrating torso injuries. $\mathrm{N}$ Engl J Med. 1994;331:1105-1109.

[8] Mizushima Y, Nakao S, Idoguchi K, et al. Fluid resuscitation of trauma patients: How much fluid is enough to determine the patient's response? Am J Emerg Med. 2017;35:842-845.

[9] Broder G, Weil M H. Excess Lactate: An Index of Reversibility of Shock in Human Patients. Science. 1964;143:1457-1459.

[10] Broussard CL. Hyperbaric Oxygenation and Wound Healing. J Wound Ostomy Continence Nurs. 2003; 30:210-216. 
[11] Boykin JV. Hyperbaric oxygen therapy: a physiological approach to selected problem wound healing. Wounds 1996;8:183-198.

[12] Dübendorfer C, Billeter AT, Seifert B, et al. Serial lactate and admission SOFA scores in trauma: an analysis of predictive value in 724 patients with and without traumatic brain injury. Eur J Trauma Emerg Surg. 2013;39:25-34

[13] Dula DJ, Wood GC, Rejmer AR, et al. Use of prehospital fluids in hypotensive blunt trauma patients. Prehosp Emerg Care. 2002;6:417-420.

[14] Duke MD, Guidry C, Guice J, et al. Restrictive fluid resuscitation in combination with damage control resuscitation: time for adaptation. J Trauma Acute Care Surg. 2012;73:674-678.

[15] Aslar AK, Kuzu MA, Elhan AH, et al. Admission lactate level, and the APACHE II score are the most useful predictors of prognosis following torso trauma. Injury. 2004;35:746-752.

[16] Sammour T, Kahokehr A, Caldwell S, et al. Venous glucose and arterial lactate as biochemical predictors of mortality in clinically severely injured trauma patients a comparison with ISS and TRISS. Injury. 2009;40:104-
108.

[17] Meregalli A, Oliveira RP, Friedman G. Occult hypoperfusion is associated with increased mortality in hemodynamically stable, high-risk, surgical patients. Crit Care. 2004;8:60-65.

[18] Uzoigwe CE, Venkatesan M, Smith R, et al. Serum lactate is a prognostic indicator in patients with hip fracture. Hip Int. 2012;22:580-584.

[19] Lewis CT, Naumann DN, Crombie N, et al. Prehospital point-of-care lactate following trauma: A systematic review. J Trauma Acute Care Surg. 2016;81:748-755.

How to cite this article: Cengiz Güney, Abuzer Coskun. The Effect of Lactate Level on Early Mortality and Late Wound Healing in Children with Thoracoabdominal Trauma with Fluid Treatment Before Hospital. Signa Vitae. 2020;16(2):75-81. doi:10.22514/sv.2020.16.0068. 